\title{
USO DA TÉCNICA CFD PARA VERIFICAR O DESEMPENHO DO LEITO FLUIDIZADO COM DIFERENTES RAZÕES DE ASPECTO (HE/DI)
}

\author{
Ana Paula Silva $\operatorname{Artur}^{1}$ (anaps.artur@gmail.com), Elaine Cristina Batista da Silva ${ }^{2}$ \\ (ellainecristinaa@hotmail.com), Welberth Santos Laizo ${ }^{3}$ (welbs@ msn.com), Reimar de Oliveira Lourenço ${ }^{4}$ \\ (reimar@ufsj.edu.br) \\ (1) Universidade Federal de São João del Rei (UFSJ) - Programa de Pós-Graduação em Engenharia Química - Ouro Branco - MG \\ (2) Universidade Federal de São João del Rei (UFSJ) - Departamento de Engenharia Química (DEQUI) - Ouro Branco - MG \\ (3) Universidade Federal de São João del Rei (UFSJ) - Departamento de Engenharia Química (DEQUI) - Ouro Branco - MG \\ (4) Universidade Federal de São João del Rei (UFSJ) - Departamento de Engenharia Química (DEQUI) - Ouro Branco - MG
}

RESUMO: Leitos fluidizados são amplamente utilizados em processos industriais, e a simulação computacional vem ganhando espaço no seu planejamento e uso. O presente trabalho objetivou estudar o escoamento no interior de um leito fluidizado, justificado pelo por diminuir custos de desenvolvimento e pesquisa durante o estudo de desempenho do equipamento, bem como possibilitar a sua otimização. Uma geometria $3 D$, do equipamento, foi gerada com as dimensões $\operatorname{lm} \times 0,2 m \times 0,1 m$, utilizando espaçamento de malha 0,01 " mesh" para o corpo do reator e um refino para 0,005 "mesh", na entrada. Utilizou-se esferas de vidro como parte sólida e ar como fluido de transporte. Foi possível verificar o desenvolvimento do escoamento através do leito, passando do estado de leito fixo até a fluidização turbulenta e velocidade de mínima fluidização de $0,035 \mathrm{~m} / \mathrm{s}$, representando resultado próximo aos encontrados na literatura com tempo computacional satisfatório. Esses dados preditivos são de suma importância na estimativa de econômica no projeto e construção desses equipamentos.

PALAVRAS-CHAVE: $C F D$, Perfil da dinâmica do escoamento, leito fluidizado.

\section{INTRODUÇÃO}

Leitos fluidizados são amplamente utilizados em várias aplicações industriais entre elas a secagem de partículas, recobrimento e granulação de sólidos, sínteses e reações catalíticas, regeneração catalítica, combustão e gaseificação de carvão (CREMASCO, 2012), devido às suas excelentes propriedades de mistura e transferência de calor e massa interfacial. (WU; DE MARTÍN; COPPENS, 2017).

Para se conquistar o objetivo desejado, e ter uma maximização na eficiência com segurança, deve-se escolher o modelo que seja adequado no contato gás-sólido no leito. Normalmente a fluidização se enquadra numa dessas classes: fluidização homogênea ou borbulhante (NITZ; GUARDANI, 2008).

A fluidização homogênea está presente em sistemas liquido-sólido e gás-sólido, ela ocorre quando a velocidade do gás varia da mínima fluidização até a velocidade mínima de ebulição, esse

$$
\begin{array}{r}
\text { IX COEN - Congresso de Engenharias da UFSJ } \\
\text { Interconexão. }
\end{array}
$$


processo é caraterístico por se ter as partículas distribuídas uniformemente ao longo do fluido, e não apresentar formação de aglomerados e bolhas (ZHANG et al., 2008).

A Fluidização borbulhante, ocorre no sistema gás-sólido, quando o sistema em questão se encontra em velocidade superficial do gás maior que a velocidade mínima de borbulhamento, nesse caso, diferentemente do sistema acima, a distribuição de partículas no gás não é uniforme, logo se tem bolhas de gás com baixo teor de sólidos e uma fase de partículas densas separadas (ZHANG et al., 2008).

A Fluido Dinâmica Computacional (CFD) vem ganhando espaço e está se tornando um ótimo método para explorar a dinâmica complicada de fluidos no leito fluidizado. Esses modelos numéricos de fluxos de partículas-fluidos podem ser classificados de acordo com o tratamento das fases particulada e fluida: sendo estes métodos Euleriano-Euleriano e Euleriano-Lagrangeano (WU et al., 2016).

No método Euleriano-Euleriano, as duas fases são tratadas como fases contínuas interpenetradas, na qual ambas as fases são descritas através de um conjunto de equações de conservação que possuem termos em forma de interação (DUARTE; MURATA; BARROZO, 2008).

No modelo Euleriano-Lagrangeano a fase fluida é tratada como uma fase contínua, sendo as equações de conservação de massa, momento e energia resolvidas para esta fase, enquanto a fase sólida é tratada como uma fase discreta, onde cada partícula individual é rastreada pela integração de todas as forças atuantes sobre ela, essas forças incluem forças de interação entre as partículas, bem como interação de partículas com fase contínua (DUARTE; MURATA; BARROZO, 2008). O que dificulta a utilização desse modelo é que o mesmo necessita de alta capacidade computacional para rastreamento das partículas, e, por causa dessa limitação ele tem sua aplicação em problemas de pequena ou média escala (IQBAL; RAUH; DELGADO, 2015).

Segundo Santos (2011), o modelo Euleriano deve ser aplicado quando a gravidade atua de forma relevante na separação entre as fases, e essa é presente em equipamentos como colunas de bolha, risers, suspensão de partículas, leitos fluidizados e leito de jorro, dentre outros.

Em busca de melhorar e promover a expansão de processos, deve-se buscar a compreensão da hidrodinâmica dos leitos fluidizados. No entanto, demonstrou-se um desafio a coleta de dados experimentais com um bom grau de detalhamento, e essa dificuldade é ainda mais perceptível para 
sistemas tridimensionais (3D), principalmente nos casos em larga escala, isto ocorre devido à falta de acesso óptico e ao elevado esforço computacional exigido para esse procedimento. Devido a esses empecilhos as simulações computacionais de grande escala de leitos fluidizados ocorrem em sistemas bidimensionais (2D ou pseudo-2D). Mas com a evolução tecnológica teve-se o crescimento na disponibilidade de poder computacional e esquemas numéricos mais eficientes, consequentemente as simulações de leitos fluidizados em 3D veio a se tornar realidade (YANG et al., 2017).

O presente estudo propõe investigar o uso do CFD no leito fluidizado em 3D, afim de obter maiores conhecimentos a respeito da ferramenta computacional, visto que seu uso pode promover a reduções de custo e tempo de projeto, quando aplicadas adequadamente.

\section{OBJETIVO}

\section{2 .1 Objetivo Geral}

A pesquisa desenvolvida neste trabalho teve como objetivo estudar, através de simulações via CFD, o escoamento no interior de um leito fluidizado em 3D de um sistema gás-sólido e, a partir dos dados obtidos gerar as curvas característica do leito, discutir os perfis de porosidade e analisar as características particulares da simulação para determinada partícula e conformação do leito.

\section{2 .1 Objetivo Específico}

I. Verificar o escoamento fluidodinâmico usando o modelo de arraste Syamlal-Obrien apresentará o escoamento desejado;

II. Fazer verificação de malha aplicada ao leito;

III. Avaliação dos parâmetros correlacionados ao processo, (velocidade de mínima fluidização, queda de pressão e porosidade do leito);

IV. Verificar se o incremento de tempo adotado, proporcionará resultados satisfatórios;

\section{REFERENCIAL TEÓRICO}

As equações de conservação são escritas para ambas as fases, o que dá origem a um sistema de equações similares, facilitando a manipulação matemática do sistema. A descrição adequada das forças interfaciais, presentes nas equações de balanço de quantidade de movimento de ambas as fases, 
é de suma importância na precisão das simulações, sendo a força de arraste Equação 1 a principal força que age sobre as partículas (SANTOS, 2011), a força de arraste correspondente a transferência de momento na interface entre as fases gasosa e sólida sendo uma das forças dominantes nos balanços de momento (TAGHIPOUR; ELLIS;WONG, 2005). A força de arraste exercida sobre as partículas em sistemas líquidos-sólidos é representada a diante:

$$
\vec{f}_{\text {arraste }}=K_{f s}\left(\vec{v}_{s}-\vec{v}_{f}\right)
$$

Essa força entre as partículas e o fluido desempenha um papel essencial na predição da estrutura de fluxo de fluidização de um leito. Muitos modelos de arrasto têm sido desenvolvidos para calcular a troca dinâmica interfase em leito fluidizado, tais como modelos de arrasto (WEN; YU, 1966), (SYAMLAL; O’BRIEN, 1989), e (GIDASPOW et al.,1992), etc (LAN et. al., 2014).

Para sistemas diluídos um modelo apropriado é o de (WEN; YU, 1966), onde o coeficiente de troca gás sólido é definido pelas Equação 2 e Equação 3:

$$
\begin{aligned}
& K_{f S}=\frac{3}{4} C_{D} \frac{\alpha_{s} \alpha_{f} \rho_{f}\left|\vec{v}_{s}-\vec{v}_{f}\right|}{d_{S}} \alpha_{f}^{-2,65} \\
& C_{D}=\frac{24}{\alpha_{f} R e_{S}}\left[1+0,15\left(\alpha_{f} R e_{s}\right)^{0,687}\right]
\end{aligned}
$$

Syamlal e O’brien (1989), propuseram um modelo onde a função de arraste tem a seguinte forma apresentada na Equação 4:

$$
C_{D}=\left(0,63+\frac{4,8}{\sqrt{R e_{s} / v_{r, s}}}\right)^{2}
$$

Onde o coeficiente de troca sólido-fluido Equação 5 tem a seguinte forma.

$$
K_{f s}=\frac{3 \alpha_{s} \alpha_{f} \rho_{f}}{4 v_{r, s}^{2} d_{s}} C_{D}\left(\frac{R e_{s}}{v_{r, s}}\right)\left|\vec{v}_{s}-\vec{v}_{f}\right|
$$

O modelo de arraste de Syamlal (ZHONG, et. al., 2014) para partícula-partícula foi obtido utilizando os métodos da teoria cinética dos gases densos. O modelo é expressado na Equação 6.

$$
K_{s a}=\frac{3\left(1-e_{s a}\right)\left(\frac{\pi}{2}+C_{f r, s a} \frac{\pi^{2}}{8}\right) \rho_{s} \alpha_{s} \alpha_{s} \rho_{a}\left(d_{a}+d_{s}\right) g_{0, a s}}{2 \pi\left(\rho_{a} d_{a}^{3}+\rho_{s} d_{s}^{3}\right)}\left|\vec{v}_{s}-\vec{v}_{a}\right|
$$




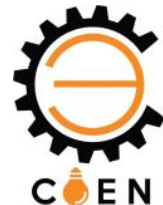

A porosidade do leito $(\varepsilon)$ pode ser obtida fazendo-se a subtração entre a unidade e a fração de sólidos $\left(\varepsilon_{s}\right)$. Esta relação é mostrada pela Equação 7.

$$
\varepsilon=1-\varepsilon_{s}
$$

Na simulação foi utilizada uma fração de sólidos $\varepsilon_{s}$ de 0,6 e para tal efeito têm-se a porosidade de leito fixo $\varepsilon_{f}$ de 0,4 (adimensional).

Para uma maior economia de tempo e capacidade de processamento é interessante obter um número reduzido de equações resolvidas, sendo uma abordagem interessante a de Euler para leitos fluidizados (HAMZEHEI, 2014).

O modelo Euleriano, denominado Modelo de Dois Fluidos é considerado o modelo mais adequado para a simulação de escoamentos gás-sólido, descrevendo o comportamento das duas fases como meios contínuos interpenetrantes coexistindo em um mesmo domínio (MARTINS, 2015).

A abordagem do modelo Euler-Euler, considera as fases contínua e interpenetrante, ou seja, não há vazios ou espaços e não tem espaço definido para cada fase. A fração volumétrica $\alpha$, quantifica cada fase no volume de controle e por definição o somatório das frações é igual a um Equação 8.

$$
\alpha_{\mathrm{s}}+\alpha_{\mathrm{g}}=1
$$

Para a caracterização da conservação de massa temos a Equação 9 e Equação 10:

$$
\begin{aligned}
& \frac{\partial}{\partial t}\left(\alpha_{g} \rho_{g}\right)+\nabla\left(\alpha_{g} \rho_{g} \vec{v}_{g}\right)=\sum_{p=1}^{n} \dot{m}_{p q}-\alpha_{q} \frac{d_{q} \rho_{q}}{d t} \\
& \frac{\partial}{\partial t}\left(\alpha_{s} \rho_{s}\right)+\nabla\left(\alpha_{s} \rho_{s} \vec{v}_{s}\right)=\sum_{g=1}^{n}\left(\dot{m}_{f s} \dot{m}_{s f}\right)+S_{s}
\end{aligned}
$$

Sendo $\dot{m}$ a taxa de transferência de massa, S o termo de fonte de massa. Como não se considerada a transferência de massa entre as fases, o lado direito das Equações 9 e 10 são nulos.

A Equação 11 de transporte de momentum para a fase gasosa é:

$$
\begin{aligned}
& \frac{\partial}{\partial t}\left(\alpha_{f} \rho_{f} \vec{v}_{f}\right)+\nabla\left(\alpha_{f} \rho_{f} \vec{v}_{f} \vec{v}_{f}\right)=-\alpha_{f} \nabla p+\nabla \cdot \overline{\overline{\tau_{f}}}+\alpha_{f} \rho_{f} \vec{g}+\sum_{p=1}^{n}\left(\vec{K}_{f s}\left(\vec{v}_{s}-\vec{v}_{f}\right)-\dot{m}_{f s} \vec{v}_{f s}\right) \\
& +\alpha_{f} \rho_{f}\left(\vec{F}_{f}+\vec{F}_{l i f t, f}+\vec{F}_{v m, f}\right)
\end{aligned}
$$

Onde $\vec{g}$ é a gravidade, $\overrightarrow{F_{f}}$ é a força de campo, $\vec{F}_{l i f t, f}$ é a força de ascensão que atua na partícula, principalmente devido aos gradientes de velocidade no campo do escoamento da fase primária (f). 

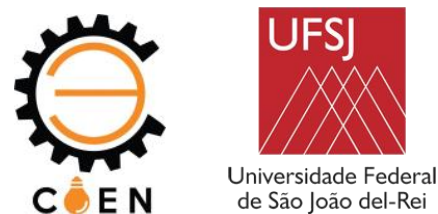

Esta força de ascensão é a mais significativa para partículas maiores. A força de massa virtual é representada por e $\vec{F}_{v m, f}$ a velocidade na interface é representada por $\vec{v}_{f s}$. A velocidade na interface está relacionada com a transferência de massa, por exemplo, se há transferência de massa da fase sólida para a fase fluida então $\vec{v}_{f s}=\vec{v}_{s}$. No entanto se há transferência de massa da fase fluida para a fase sólida então $\vec{v}_{f s}=\vec{v}_{f}$.

O tensor tensão da fase gasosa é dado ela Equação 12:

$$
\overline{\overline{\tau_{g}}}=\rho_{g} \mu_{g}\left(\nabla \vec{v}_{g}+\nabla \vec{v}_{g}^{T}\right)+\alpha_{g}\left(\lambda_{g}-\frac{2}{3} \mu_{g}\right) \nabla \vec{v}_{g} \overline{\bar{I}}
$$

A Equação 13 de transporte de momentum para a fase sólida é:

$$
\begin{aligned}
& \quad \frac{\partial}{\partial t}\left(\alpha_{s} \rho_{s} \vec{v}_{s}\right)+\nabla\left(\alpha_{s} \rho_{s} \vec{v}_{s} \vec{v}_{s}\right)=-\alpha_{s} \nabla p+\nabla \cdot \overline{\bar{\tau}}_{s}+\alpha_{s} \rho_{s} \vec{g}+\sum_{f=1}^{n}\left(K_{f s}\left(\vec{v}_{f}-\vec{v}_{s}\right)+\dot{m}_{f s} \vec{v}_{f s}\right) \\
& +\alpha_{s} \rho_{s}\left(\vec{F}_{s}+\vec{F}_{l i f t, s}+\vec{F}_{v m, s}\right)
\end{aligned}
$$

\section{MATERIAIS E MÉTODOS}

A simulação foi executada em um computador com configurações: Processador Intel ${ }^{\circledR}$ Core ${ }^{\mathrm{TM}}$ i5, 8 GB de memória RAM, HD de 1 TB e sistema operacional de 64 Bits. A estratégia de simulação adotada para o presente trabalho, face a capacidade de processamento disponível, foi a simulação tridimensional (3D), com uso de um pacote comercial de CFD que usa a técnica de Volumes Finitos para o processamento numérico do problema. O método de volumes finitos baseia-se em dividir um volume de controle em vários volumes de controle menores, até quase atingir uma dimensão que torne as equações a serem resolvidas dentro deste volume como uma função de ponto, sendo resolvida de forma iterativa ou simultaneamente todo o conjunto de equações diferenciais obtidas. Este método torna as equações governantes discretizadas dentro do domínio computacional (espaço tempo) (BLANCO, 2013).

O leito 3D foi construído com as dimensões $1 \mathrm{~m} \times 0,2 \mathrm{~m} \times 0,1 \mathrm{~m}$. A malha utilizada foi a estruturada, com 10.153 nós e 8.400 elementos, escolhida por preencher de forma satisfatória os espaços da geometria em questão, ela foi refinada com mesh 0,01 para o corpo do reator e na parte inferior onde se encontra a entrada do leito, região de interesse, foi realizado um refino maior, 0,005 


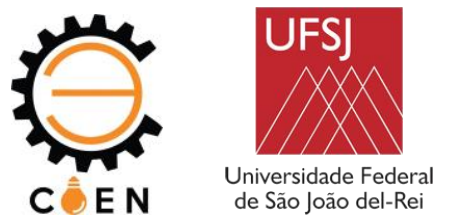

mesh, utilizando para tal, uma esfera de influência de raio $0,3 \mathrm{~m}$ partindo do centro da base, conforme mostrado na Figura 1.
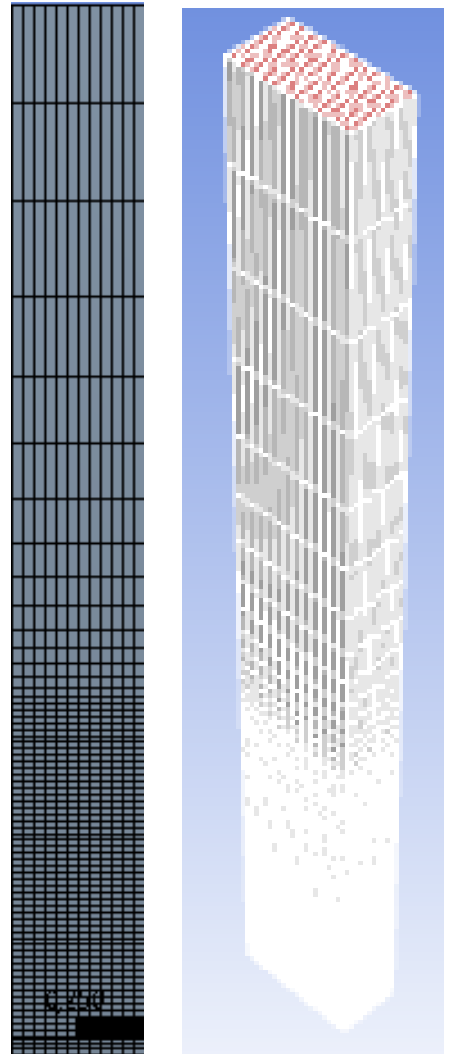

Figura 1: Configuração da malha aplicada a geometria. Fonte: Autores, 2019.

O material utilizado na simulação foram esferas de vidro com $125 \mu \mathrm{m}$ de diâmetro, esfericidade 1 e densidade de $2500 \mathrm{~kg} / \mathrm{m}^{3}$. E o fluido de transporte foi o ar. A desfluidização foi realizada para as velocidades de $0,20 \mathrm{~m} / \mathrm{s}$ a $0,02 \mathrm{~m} / \mathrm{s}$.

Adotou-se o modelo multifásico euleriano, que trata gás e partículas como um contínuo interpenetrante. Esta abordagem numérica minimiza o custo computacional, principalmente para fluxos com alta concentração de partículas, e também considera os efeitos da interação entre as duas fases. Como o volume de uma fase não pode ser ocupado pela outra, o conceito de fração de volume de fase é introduzido, no qual no projeto em questão, usou-se $40 \%$ de ar e $60 \%$ de particulado, estes valores podem variar, contudo foram escolhidos por proporcionarem a altura mínima de fluidização. Essas frações de volume são assumidas como funções contínuas de espaço e tempo. Sendo que as leis 

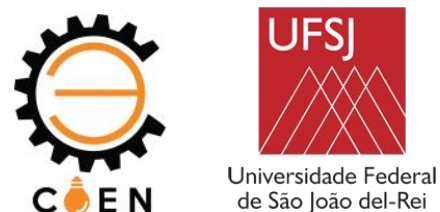

de conservação de massa e momento devem ser satisfeitas para cada fase individualmente (DUARTE; MURATA; BARROZO, 2008).

O critério de convergência e o time step são aplicados no software para que os mesmos retratem a realidade do escoamento no equipamento. Estudos indicam que resíduos acima de $10^{-4}$ são muito pouco confiáveis e servem apenas para fornecer uma ideia superficial sobre o escoamento, exatamente por essa informação aplicou-se no estudo o critério de convergência e time step de $10^{-3}$ (CÓSTOLA 2011).

As condições e modelos utilizados no programa estão indicados na Tabela 1 abaixo:

Tabela 1: Condições adotadas na simulação. Fonte: Autores, 2019.

\begin{tabular}{|cc|}
\hline Modelo & Euleriano Multifásico: (Implícito) \\
\hline Interação fluido-partícula & Syamlal-Obrien \\
\hline Viscosidade Cinética & Syamlal-Obrien \\
\hline Viscosidade Granular & Lun-et-al \\
Pressão dos sólidos & Syamlal-Obrien \\
Distribuição Radial & Lun-et-al \\
\hline Altura de preenchimento do leito & $0,2 \mathrm{~m}$ \\
\hline Fração de volume dos sólidos & 0.6 \\
\hline Critério de Convergência & $1 \times 10^{-3}$ \\
Time Step & $1 \times 10^{-3} \mathrm{~s}$ \\
Máximo de iterações & 200 \\
& Pressão $-0,2$ \\
Parâmetros de Relaxação & Densidade -1 \\
& Força de Corpo -1 \\
& Temperatura Granular $-0,2$ \\
& Fração Volumétrica $-0,2$ \\
& Momento $-0,2$ \\
\hline
\end{tabular}

A simulação foi realizada inserindo os dados no software e a partir daí variou-se a entrada de ar até que se obtivesse as características desejadas para o leito.

Os gráficos e comparativos (perfis de porosidade e velocidade) foram elaborados a partir da captura de frames do leito em determinados pontos ou velocidades. 

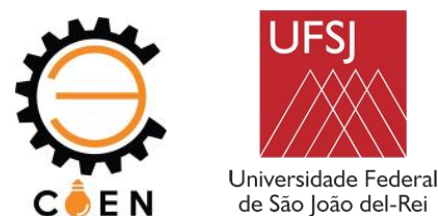

\section{RESULTADOS E CONCLUSÕES}

A análise dos resultados obtidos foi realizada através dos gráficos de perfis de porosidade, queda de pressão e curva característica do leito, cada minuto físico de simulação levou em média duas horas e meia para que as iterações fossem concluídas. Totalizando ao final do estudo 80 horas de simulação computacional, os resultados seguem abaixo:

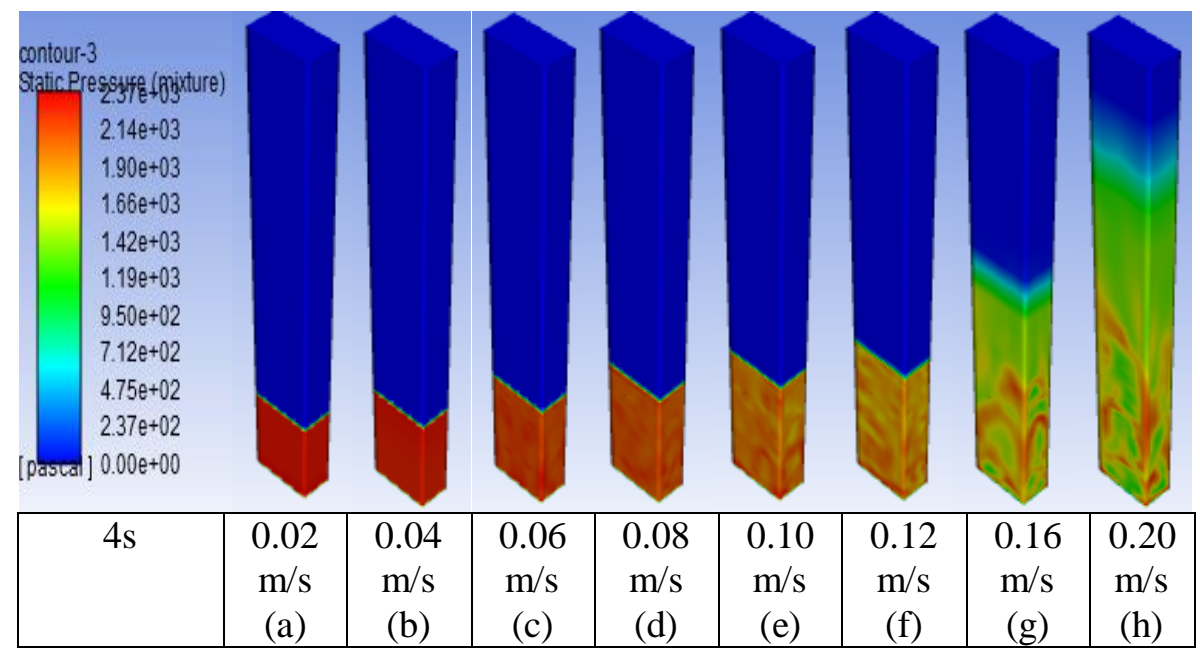

Figura 2: Perfis de porosidade do leito no tempo de $4 \mathrm{~s}$ para diferentes vazões de ar. Fonte: Autores, 2019.

Quando o fluido ascende ao leito de partículas com uma velocidade incapaz de causar-lhe movimento, Figura 2 (a), temos o leito fixo, nesse instante o fluido percola os espaços entre as partículas estáticas. Com o aumento da velocidade do gás entrante, começa-se a observar uma pequena movimentação e expansão das partículas no leito, e há um constante aumento da queda de pressão. Conforme esta vazão de gás vai sendo aumentada, as forças atuantes no leito (arraste, empuxo e peso), tendem a se igualar, e as partículas começam a oferecer menor resistência a fluidização adquirindo características de fluido, nesse ponto, temos o leito fluidizado Figura 2 (b, c, d, e, f), onde há condições favoráveis à transferência de calor e massa, indispensáveis para as diversas operações para o qual o leito é indicado, a queda de pressão a partir desse momento permanece constante. Incrementos na vazão de gás acima da velocidade mínima de fluidização resultam em uma progressiva expansão das partículas em direção ao topo do leito, gerando grandes instabilidades, formação de bolhas e canais preferenciais de passagem, tendo assim, o leito borbulhante Figura 2 (g 


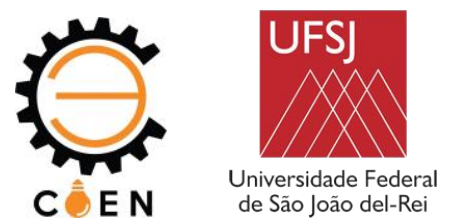

e h). Se for persistido o aumento na vazão, teremos o transporte pneumático, que não é o desejado nesse sistema.

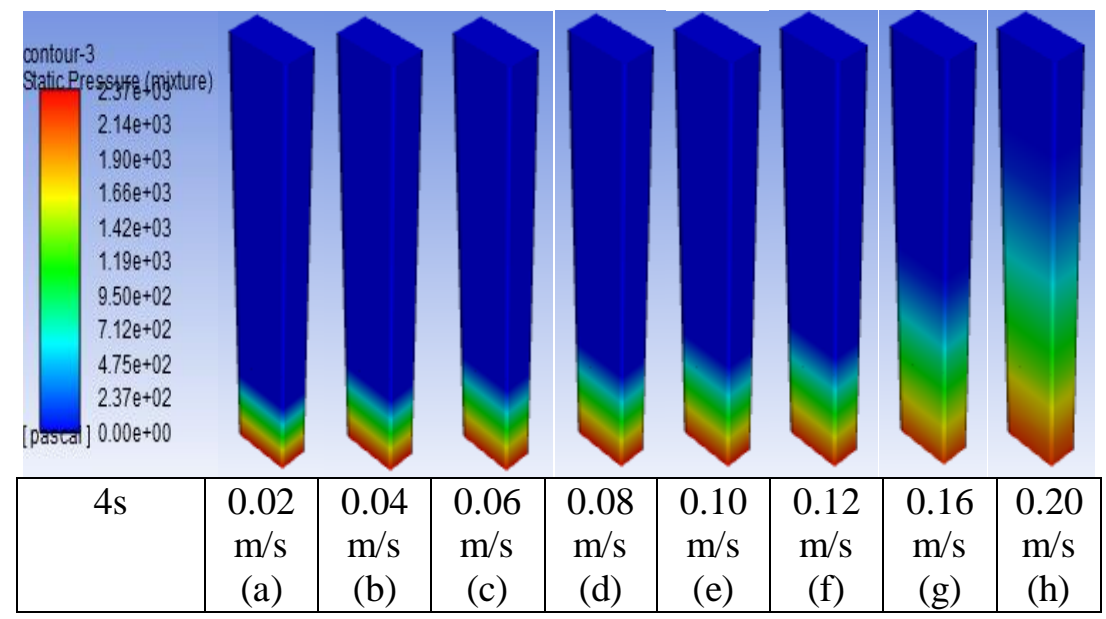

Figura 3: Perfis de pressão do leito no tempo de 4s para diferentes vazões de ar. Fonte: Autores, 2019.

A queda de pressão no leito se concentra preferencialmente na sua base, onde as partículas se encontram inicialmente e onde o esforço para coloca-las em movimento é maior.

O aumento na queda de pressão é diretamente proporcional ao aumento na velocidade do gás entrante, até que se atinja a velocidade de mínima fluidização Figura 3 (b), nesse ponto a queda de pressão é superada e tende a manter-se constante Figura 3 (c, d, e, f), porém, se o aumento da vazão for progressivo para além dessa velocidade, a queda de pressão tende a aumentar novamente, Figura $3(\mathbf{g}, \mathbf{h})$.

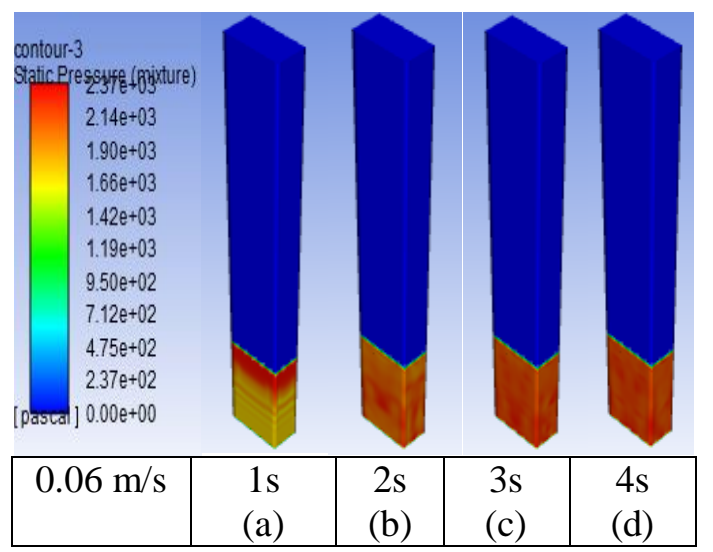

Figura 4: Perfis de porosidade do leito para a mesma velocidade em tempos diferentes de simulação. Fonte: Autores, 2019. 


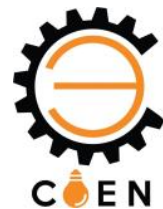

$\mathrm{Na}$ Figura 4 (a) temos as primeiras vazões de ar entrante na velocidade de $0,06 \mathrm{~m} / \mathrm{s}$ o que faz com o que as partículas no leito sejam, num primeiro momento, injetadas para cima.

A partir de 2 segundos físicos de simulação em diante Figura 4 (b, c, d), tem-se a homogeneidade e constância das partículas no leito.
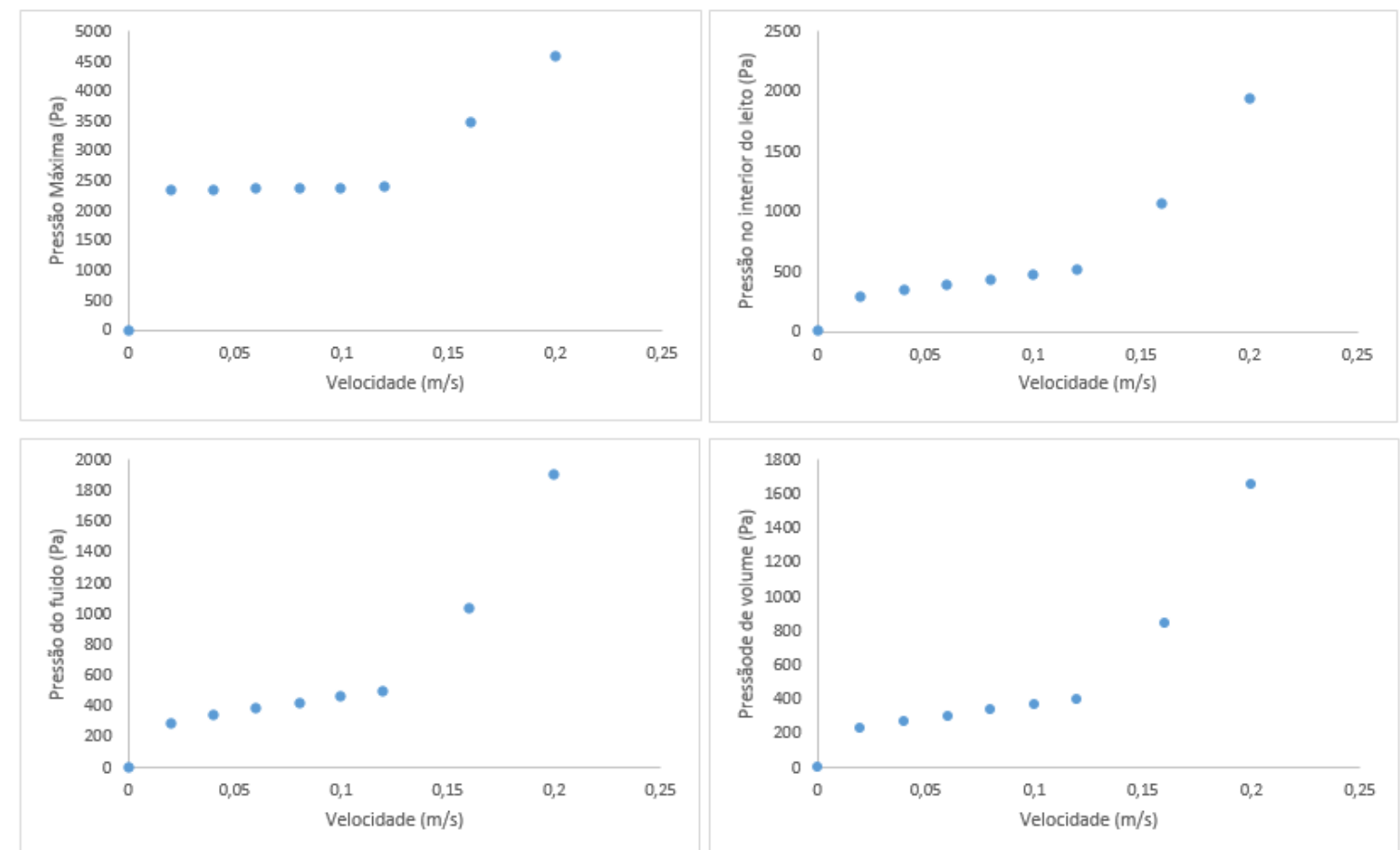

Figura 5: Gráficos de queda de pressão versus velocidade do ar de entrada. Fonte: Autores, 2019.

Os gráficos acima representados pela Figura 5 se referem a dados de queda de pressão obtida através da Área Média (Área), da Entrada de Ar (Entrada), Interior do Reator (Interior) e, do Volume Médio (Volume), sendo os três primeiros obtidos através de integrais de superfície e o último, integral de volume, todos estes dados foram fornecidos pelo próprio software, e corroboram ao que foi já dito anteriormente, que a queda de pressão tende a aumentar quando se tem o aumento da velocidade.

A partir da análise da Figura 5 é possível perceber que não há grande variação nas características iniciais do sistema, apenas quando este começa a se tornar turbulento existe uma grande variação na leitura dos dados. O que era esperado devido ao movimento caótico como característica primária quando o sistema toma o regime turbulento como padrão de ação. 

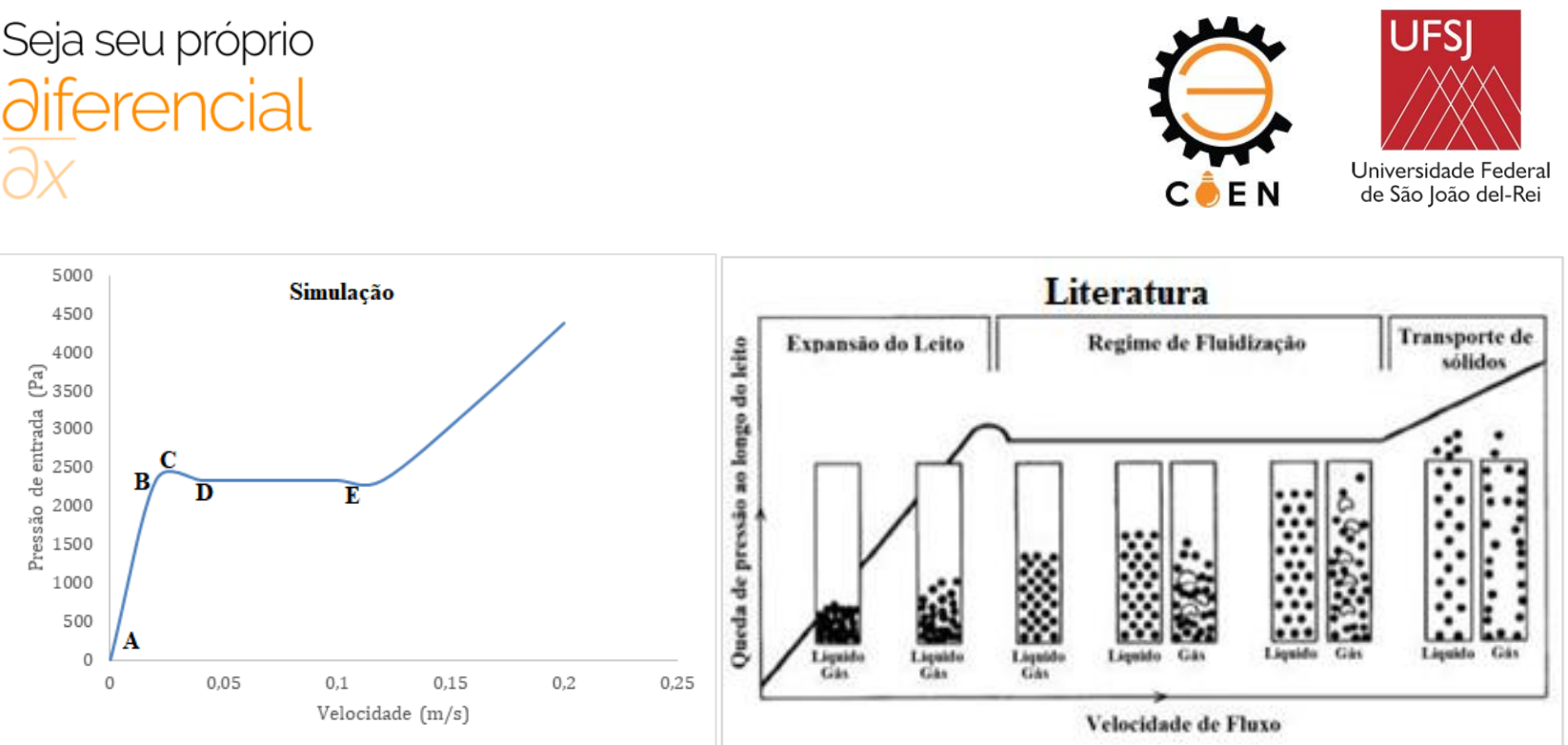

Figura 6: Curva Característica do Leito.

Simulação. Fonte: Autores, 2019

Literatura. Fonte: (BORGES, 2017)

A curva característica do leito Figura 6 é um método experimental que permite determinar a Velocidade de Mínima Fluidização, ela relaciona a queda de pressão à velocidade do ar de entrada. Interpretando o gráfico pode-se inferir a velocidade de mínima fluidização de $0,035 \mathrm{~m} / \mathrm{s}$ frente a uma queda de pressão na mínima fluidização de $2 \mathrm{KPa}$, esses valores são obtidos através da interseção da linha do leito fixo com a linha horizontal correspondente a região de fluidização do material.

Comparando a Simulação com a Literatura (BORGES, 2017), Figura 6, nota-se à similaridade. A região compreendida pelo intervalo $\mathrm{AB}$ pode ser dita como leito fixo, ou estático, as partículas nessa região estão bem acomodadas e o regime é quase sempre laminar. No ponto B a perda de carga é igual ao peso dos sólidos, nesse ponto o leito se encontra quase em repouso contendo características de um fluído e é possível observar a fluidez do leito, além disso, neste setor as partículas mudam de posição e rearranjam-se. Já no ponto C ocorre a mínima fluidização, ou seja, há o início da fluidização. No intervalo compreendido pela curva $\mathrm{CD}$, indica o movimento desordenado das partículas com frequentes choques devido ao aumento da porosidade e menor perda de carga junto com o aumento da velocidade. No intervalo correspondido por AD o leito é dito em expansão. No ponto $\mathrm{D}$ a perda de carga começa a ficar constante, não ocorrendo o contato entre as partículas. No intervalo DE a velocidade varia linearmente com a queda de pressão até chegar no ponto E, nesse intervalo ocorre a fluidização. No ponto E as partículas começam a ser carregadas pelo fluído e perdese a funcionalidade do sistema, nessa região a fluidização é dita contínua ou em fase diluída., a partir daí ocorre a fase chamada de transporte pneumático. 

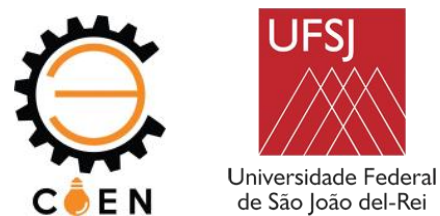

O gráfico de pressão versus velocidade superficial é bastante utilizado como indicação da qualidade da fluidização, especialmente quando a observação visual não é possível. A velocidade de mínima fluidização é especifica para determinada conformação do leito, particularidades como dimensões do leito e da partícula influenciam diretamente nesta velocidade, o que faz com que cada sistema seja único para determinada característica.

Conclui-se pelo presente estudo que a simulação realizada permitiu inferir a velocidade de mínima fluidização de $0,035 \mathrm{~m} / \mathrm{s}$ frente a uma queda de pressão na mínima fluidização de $2 \mathrm{KPa}$, para o sistema gás-sólido (ar-esfera de vidro). Além de que, os perfis de porosidade permitiram identificar os diversos estágios do processo de fluidização e inferir a velocidade na qual o leito se fluidiza, coincidindo com o método experimental da curva característica. Os valores obtidos estão próximos aos encontrados na literatura, indicando que o modelo de arraste e a malha utilizada no estudo se adequaram ao equipamento, e pelo tempo computacional gasto para a obtenção dos dados demonstrou-se satisfatório.

$\mathrm{O}$ incremento de tempo usado na simulação foi de $10^{-3} \mathrm{~s}$, e neste passo de tempo (Time Step) nas velocidades mais altas observou-se uma exigência alta do equipamento, devido a simulação estar sendo realizada no modelo tridimensional - 3D, como os resultados quando se avalia os frames e os gráficos já foram satisfatórios, não teve necessidade de ocorrer a diminuição do time step para $10^{-4} \mathrm{~s}$.

Esses dados preditivos são de suma importância para os projetos e construções de leito, visto que indicam os parâmetros e condições ideias para seu funcionamento mediante conformações específicas, antes de sua implementação física.

\section{DIREITOS AUTORAIS.}

Os autores são os únicos responsáveis pelo conteúdo das informações contidas neste artigo.

\section{REFERÊNCIAS}

BLANCO, R. Análise De Uma Câmara De Combustão Em Escala Piloto Através De Fluidodinâmica Computacional. Trabalho de Graduação em Engenharia Mecânica. Faculdade de Engenharia de Guaratinguetá. Universidade Estadual Paulista, 65 p., 2013.

BORGES, P. W. S. Readequação da prática de laboratório de Engenharia Química 1: Leito Fluidizado. Trabalho de Graduação. Faculdade de Engenharia Química. Universidade Federal de 

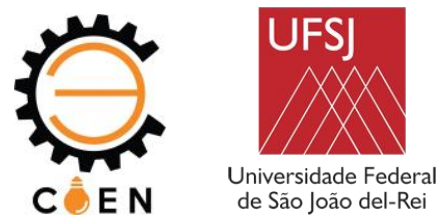

Uberlândia, 34 p., 2017.

CÓSTOLA, D.; PEINADO, M. A. Aplicação de CFD para o cálculo de coeficientes de pressão externos nas aberturas de um edifício,2011.

CREMASCO, M. A. Operações unitárias em sistemas particulados e fluidomecânicos. São Paulo: Blucher, v. 212, p. 423, 2012.

DUARTE, C. R.; MURATA, V. V.; BARROZO, M. A. S. Experimental and numerical study of spouted bed fluif dynamics. Brazilian Journal of Chemical Engineering, v. 25, n. 1, p. 95-107, mar. 2008.

GIDASPOW, D.; BEZBURUAH, R.; DING. J. Hydrodynamics of circulating fluidized beds, kinetic theory approach in fluidization, In: Proceedings of the 7th Engineering Foundation Conference on Fluidization, 1992, p. 75-82.

IQBAL, N.; RAUH, C.; DELGADO, A. Numerical simulations of particle-laden jet/spout flows using Eulerian-Lagrangian approach. Procedia Engineering, v. 102, p. 867-876, 2015.

HAMZEHEI, M. CFD Modeling and Simulation of Hydrodynamics in a Fluidized Bed Dryer with Experimental Validation. Mechanical Engineering, v. 2011, n. 2011, p. 1-8, 2014.

LAN, X.; YAN, W.; XU, C.; GAO, J.; LUO, Z. Hydrodynamics of gas-solid turbulent fluidized bed of polydisperse binary particles: Powder Technology. 2014, 262, 106 -123.

MARTINS, C. A. Simulação do Perfil Axial de Pressão na Coluna Riser de um leito fluidizado circulante. Dissertação de Mestrado, Universidade Tecnológica Federal do Paraná, 132 p., 2015.

NITZ, M.; GUARDANI, R. Fluidização Gás-Sólido - Fundamentos e Avanços. Revista Brasileira de Engenharia Química, 2008.

SANTOS, K. G. Aspectos Fundamentais Da Pirólise De Biomassa Em Leito De Jorro: Fluidodinâmica E Cinética Do Processo. Tese de Doutorado. Programa de Pós-Graduação em Engenharia Química. Universidade Federal de Uberlândia, 261 p., 2011.

SYAMLAL, M.; O'BRIEN., T. J. Computer simulation of bubbles in a fluidized bed, AIChESymp. Series, 1989, 85, 22-31.

TAGHIPOUR, F.; ELLIS, N.; WONG, C. Experimental and computational study of gas-solid fluidized bed hydrodynamics. Powder Technology. 2005, 60, 6857 -6867.

WEN, C. Y.; YU, Y. H. Mechanics of fluidization, Eng. Prog. Symp.Series, 1966, 62, 100-111. 

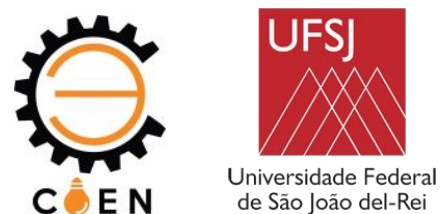

WU, K.; DE MARTÍN, L.; MAZZEI, L.; COPPENS, M. O. Pattern formation in fluidized beds as a tool for model validation: A two-fluid model based study. Powder Technology, v. 295, p. 35-42, 2016.

WU, K.; DE MARTÍN, L.; COPPENS, M. O. Pattern formation in pulsed gas-solid fluidized beds - The role of granular solid mechanics. Chemical Engineering Journal, v. 329, p. 4-14, 2017.

YANG, L.; PADDING, J. T.; BUIST, K. A.; KUIPERS, J. A. M. Three-dimensional fluidized beds with rough spheres: Validation of a Two Fluid Model by Magnetic Particle Tracking and discrete particle simulations. Chemical Engineering Science, v. 174, n. September, p. 238-258, 2017.

ZHANG, K.; BRANDANI, S.; BI, J.; JIANG, J. CFD simulation of fluidization quality in the three-dimensional fluidized bed. Progress in Natural Science, v. 18, n. 6, p. 729-733, 2008.

ZHONG, H.; LAN, X..; GAO, J.; XU, C. Effect of particle frictional sliding during collisions on modeling the hydrodynamics of binary particle mixtures in bubbling fluidized beds: Powder Technology. 2014, 254, $36-43$.

\section{USE OF THE CFD TECHNIQUE TO VERIFY FLUIDIZED BREAST PERFORMANCE WITH DIFFERENT REASONS OF ASPECT (HE / DI)}
Ana Paula Silva $\operatorname{Artur}^{1}$ (anaps.artur@gmail.com), Elaine Cristina Batista da Silva ${ }^{2}$
(ellainecristinaa@hotmail.com), Welberth Santos Laizo ${ }^{3}$ (welbs@ @msn.com), Reimar de Oliveira Lourenço ${ }^{4}$ (reimar@ufsj.edu.br)

\begin{abstract}
(2) Universidade Federal de São João del Rei (UFSJ) - Departamento de Engenharia Química (DEQUI) - Ouro Branco - MG
(3) Universidade Federal de São João del Rei (UFSJ) - Departamento de Engenharia Química (DEQUI) - Ouro Branco - MG

(4) Universidade Federal de São João del Rei (UFSJ) - Departamento de Engenharia Química (DEQUI) - Ouro Branco - MG
\end{abstract}
(1) Universidade Federal de São João del Rei (UFSJ) - Programa de Pós-Graduação em Engenharia Química - Ouro Branco - MG

ABSTRACT: Fluidized beds are widely used in industrial processes, and computer simulation has been gaining space in its planning and use. The present work aimed to study the flow inside a fluidized bed, justified by the fact that it reduces development and research costs during the study of the performance of the equipment, as well as to enable its optimization. A 3D equipment geometry was generated with dimensions Im $\times 0.2 m \times 0.1 m$, using 0.01 "mesh", spacing for the reactor body and a 0.005 "mesh" refining at the inlet. Glass beads were used as the solid part and air was used as carrier fluid. It was possible to verify the development of the flow through the bed, passing from the fixed bed state to the turbulent fluidization and minimum fluidization velocity of $0.035 \mathrm{~m} / \mathrm{s}$, representing a result close to those found in the literature with satisfactory computational time. These predictive data are of paramount importance in estimating economics in the design and construction of such equipment.

KEYWORDS: $C F D$, flow dynamics profile, fluidized bed. 